\title{
A Centralized Control Algorithm for Target Tracking with UAVs
}

\author{
Pengcheng Zhan, David W. Casbeer, A. Lee Swindlehurst \\ Dept. of Elec. \& Comp. Engineering, Brigham Young University, Provo, UT, USA, 84602 \\ Telephone: (801) 422-3930 , Fax: (801) 422-0201 \\ e-mail: pz8@ee.byu.edu,casbeer@byu.edu,swindle@ee.byu.edu
}

\begin{abstract}
Due to their wide range of practical applications, Unmanned Air Vehicles (UAVs) have recently attracted considerable attention in the research community. In this paper, we focus on their use in a simple target tracking application, and take advantage of their maneuverability to improve tracking performance. A centralized control point is used to command the headings of the UAVs in order to minimize a target localization criterion based on the Kalman filter. A practical model for the system's architecture is presented, along with simulation results that show the algorithm significantly improves estimation of the target's parameters compared with the uncontrolled system.
\end{abstract}

\section{INTRODUCTION}

The use of Unmanned Air Vehicles (UAVs) has gained considerable momentum given their success in recent military operations, and their promise for important domestic applications such as border and coast patrol, fire perimeter monitoring, search and rescue, etc. The cooperation of teams of UAVs can be used to accomplish tasks that are dangerous for human operators, and they can reduce operational costs by implementing tasks using smaller and cheaper UAVs rather than larger more expensive ones.

In this paper, we consider the use of a team of UAVs for tracking a maneuvering target, a problem that has recently received some attention in the research literature. In [1], the optimal set of UAV locations is derived based on the wellknown Cramer-Rao bound. However, the results are derived under two assumptions that may not be realistic in practice: (1) the UAVs are able to form local estimates of the target position themselves, and (2) the UAVs are equi-distant to the target. In [2], [3], [4] the trajectories of a team of mobile sensors are optimized assuming a bearings-only measurement model, and [5] extends this work by adding range measurements to the problem. However, small UAVs may have a difficult time making accurate bearing measurements given their small aperture and the fact that they are much more sensitive to wind-blown disturbances.

In our work, we assume a multistatic scenario in which a basestation, either airborne or on the ground, illuminates the target with a radar signal, and the UAVs are able to make time-delay and Doppler measurements of the target's reflected signal. The UAVs feed these measurements to the base, which is running an Extended Kalman Filter (EKF) to track the

This work was supported by the U. S. National Science Foundation under Information Technology Research Grant CCR-0313056 target's position and velocity. The base then gives heading commands to each UAV in order to minimize some function of the EKF error, and hence more accurately track the target. In effect, the maneuverability of the UAVs provide the base with an EKF whose parameters are tunable to minimize the target state estimation error. Our approach can be considered to be a special but more tractable case of the sensor management problem posed in [6].

The format of the paper will be as follows: in Section II we set up the problem and describe the EKF and the assumed dynamic models. Section III describes two algorithms for determining "optimal" UAV heading angles based on the EKF updates. Section IV presents the results of a simple simulation, and some conclusions are drawn in Section V.

\section{System ARCHitecture}

\section{A. Problem Statement}

As mentioned above, we consider a multistatic radar problem in which a set of $N$ UAVs receives the signal reflected by a mobile target illuminated by a base transmitter. We assume that the UAVs are able to form time delay $\left(\tau_{i}\right)$ and Doppler $\left(f_{i}\right)$ estimates based on these signals, and transmit these measurements back to the base.

With an auto-pilot such as that described in [7], the UAVs are capable of velocity and altitude hold. Therefore, for simplicity, we consider only a two-dimensional scenario here. We define the position of the base station as the origin for our $(x, y)$ coordinate system. Let $x, y, V_{x}$, and $V_{y}$ represent respectively the position of the target and the components of the target velocity vector in the $(x, y)$ directions. Similarly, let $x_{i}$ and $y_{i}$ denote the position of the $i^{\text {th }} \mathrm{UAV}$, and assume that each UAV is flying at the same constant speed $\mathrm{V}$, with heading angle $\psi_{i}$ measured counterclockwise from the $x$-axis. According to [8] and after appropriate scaling, we have:

$$
\begin{aligned}
\tau_{i} & =\sqrt{x^{2}+y^{2}}+\sqrt{\left(x-x_{i}\right)^{2}+\left(y-y_{i}\right)^{2}} \\
f_{i} & =\frac{V_{x} x+V_{y} y}{\sqrt{x^{2}+y^{2}}}+\frac{V_{p}}{\sqrt{\left(x-x_{i}\right)^{2}+\left(y-y_{i}\right)^{2}}}
\end{aligned}
$$

where

$$
V_{p}=\left(V_{x}-V \cos \psi_{i}\right)\left(x-x_{i}\right)+\left(V_{y}-V \sin \psi_{i}\right)\left(y-y_{i}\right) .
$$

Using all the time-delay and Doppler information sent from the UAVs, the base station runs an Extended Kalman Filter (EKF) to track the target, and at the same time sends 
back the command heading for each UAV such that the next measurement received will produce an improved estimate at the base station.

\section{B. Extended Kalman Filter}

The need for the EKF is apparent from the nonlinear measurement equations in (1) and (2). We represent the target state vector at time $k$ as $\mathbf{x}_{k}=\left[\begin{array}{llll}x & y & V_{x} & V_{y}\end{array}\right]^{T}$, and arrange the measurements into the observation vector $\mathbf{h}_{k}\left(\mathbf{x}_{k}\right)=$ $\left[\begin{array}{llllll}\tau_{1} & \ldots & \tau_{N} & f_{1} & \ldots & f_{N}\end{array}\right]^{T}$. The discrete-time system and observation models are then written as:

$$
\begin{aligned}
& \mathbf{x}_{k}=\mathbf{A} \mathbf{x}_{k-1}+\boldsymbol{\omega}_{k-1} \\
& \mathbf{z}_{k}=\mathbf{h}_{k}\left(\mathbf{x}_{k}\right)+\boldsymbol{\nu}_{k},
\end{aligned}
$$

where $\boldsymbol{\omega}_{k-1} \sim N\left(\mathbf{0}, \mathbf{Q}_{k-1}\right)$ and $\boldsymbol{\nu}_{k} \sim N\left(\mathbf{0}, \mathbf{R}_{k}\right)$ are respectively the process and measurement noise, which are assumed to be uncorrelated and Gaussian with covariances:

$$
\begin{aligned}
E \boldsymbol{\omega}_{k} \boldsymbol{\omega}_{j}^{T} & =\mathbf{Q}_{k} \delta_{k, j} \\
E \boldsymbol{\nu}_{k} \boldsymbol{\nu}_{j}^{T} & =\mathbf{R}_{k} \delta_{k, j} \\
E \boldsymbol{\omega}_{k} \boldsymbol{\nu}_{j}^{T} & =\mathbf{0} .
\end{aligned}
$$

As with the standard Kalman filter, the EKF can be divided into two stages, the time update and measurement update steps. With the time update, we propagate the target's state estimate and prediction covariance matrix as

$$
\begin{aligned}
\hat{\mathbf{x}}_{k}^{-} & =\mathbf{A} \hat{\mathbf{x}}_{k-1} \\
\mathbf{P}_{k}^{-} & =\mathbf{A} \mathbf{P}_{k-1} \mathbf{A}^{T}+\mathbf{Q}_{k-1}
\end{aligned}
$$

Once we have the measurements from the sensors, we update our estimate as:

$$
\begin{aligned}
\mathbf{K}_{k} & =\mathbf{P}_{k}^{-} \mathbf{H}_{k}^{T}\left(\mathbf{H}_{k} \mathbf{P}_{k}^{-} \mathbf{H}_{k}^{T}+\mathbf{R}_{k}\right)^{-1} \\
\hat{\mathbf{x}}_{k} & =\hat{\mathbf{x}}_{k}^{-}+\mathbf{K}_{k}\left(\mathbf{z}_{k}-\mathbf{h}_{k}\left(\hat{\mathbf{x}}_{k}^{-}\right)\right) \\
\mathbf{P}_{k} & =\left(\mathbf{I}-\mathbf{K}_{k} \mathbf{H}_{k}\right) \mathbf{P}_{k}^{-}
\end{aligned}
$$

In the above equations, $\mathbf{H}_{k}=\left(\frac{\partial \mathbf{h}_{k}}{\partial \mathbf{x}}\right)^{T}$ is a function of the UAVs' position and heading. The basic idea is to reduce the "size" of the estimate covariance in equation (12) by updating the heading of the UAVs, and hence $\mathbf{H}_{k}$. In essence we are applying an outer control loop around the Kalman filter.

\section{Target and UAV Dynamic Models}

In the simulation studies whose results we present later, we will assume for simplicity a target with a constant velocity model, which can be described by

$$
\mathbf{A}=\left[\begin{array}{cccc}
1 & 0 & \Delta_{t} & 0 \\
0 & 1 & 0 & \Delta_{t} \\
0 & 0 & 1 & 0 \\
0 & 0 & 0 & 1
\end{array}\right]
$$

where $\Delta_{t}$ is the time interval between samples. We will also assume white process and measurement noise terms, although this is not necessary either:

$$
\begin{aligned}
& \mathbf{Q}_{k}=\operatorname{diag}\left(\sigma_{x}^{2}, \sigma_{y}^{2}, \sigma_{V_{x}}^{2}, \sigma_{V_{y}}^{2}\right) \\
& \mathbf{R}_{k}=\operatorname{diag}\left(\sigma_{\tau}^{2}, \ldots, \sigma_{\tau}^{2}, \sigma_{f}^{2}, \ldots, \sigma_{f}^{2}\right)
\end{aligned}
$$

Modeling errors can be accounted for by tuning the parameters in $\mathbf{Q}_{k}$ and $\mathbf{R}_{k}$.

In a similar way, we assume that we have control over each UAV's heading at different time slots. Assuming an instantaneous response to heading commands, the UAVs will fly in a straight line with a velocity $V$ and heading $\psi_{i}$ during each time interval. The inertial position of the UAV $i$ at time $k$ is given by:

$$
\begin{aligned}
& x_{i, k}=x_{i, k-1}+V \cos \left(\psi_{i, k}\right) \cdot \Delta_{t} \\
& y_{i, k}=y_{i, k-1}+V \sin \left(\psi_{i, k}\right) \cdot \Delta_{t},
\end{aligned}
$$

where $\psi_{i, k}$ is its heading at the same time.

\section{AlgorithMS}

We present two algorithms for commanding the UAV headings. In the first, we attempt to minimize the trace of the one-step ahead prediction error covariance of the EKF. In the second, we attempt to maximize the information that the new measurements would provide. These two approaches are outlined in the sections below.

\section{A. One-Step Ahead Approach}

In this approach, we command the UAVs at time $t=k$ such that at $t=k+1$ the trace of the prediction covariance $\mathbf{P}_{k+1}^{-}$ is minimized. Mathematically, the idea is to find the vector of heading commands $\Psi_{k}=\left[\psi_{1, k} \ldots \psi_{N, k}\right]^{T}$ such that,

$$
\Psi_{k}=\underset{\Psi_{k}}{\arg \min } \operatorname{tr}\left(\mathbf{P}_{k+1}^{-}\right) \text {. }
$$

If we recall Equations (9) and (12), and make appropriate substitutions, our cost function becomes:

$$
\operatorname{tr}\left(\mathbf{P}_{k+1}^{-}\right)=\operatorname{tr}\left(\mathbf{A}\left[\left(\mathbf{P}_{k}^{-}\right)^{-1}+\mathbf{H}_{k}^{T} \mathbf{R}_{k}^{-1} \mathbf{H}_{k}\right]^{-1} \mathbf{A}^{T}+\mathbf{Q}_{k}\right) .
$$

We employ a simple first-order gradient search to minimize (19). The gradient with respect to the heading of the $i^{\text {th }} \mathrm{UAV}$ at time $k$ is given by

$$
\frac{\partial \operatorname{tr}\left(\mathbf{P}_{k+1}^{-}\right)}{\partial \psi_{i, k}}=-\operatorname{tr}\left(\mathbf{A B}^{-1} \frac{\partial \mathbf{B}}{\partial \psi_{i, k}} \mathbf{B}^{-1} \mathbf{A}^{T}\right)
$$

where,

$$
\mathbf{B}=\mathbf{P}_{k}^{--1}+\mathbf{H}_{k}^{T} \mathbf{R}_{k}^{-1} \mathbf{H}_{k} .
$$

The problem boils down to calculating $\frac{\partial \mathbf{H}_{k}}{\partial \psi_{i, k}}$. The matrix $\mathbf{H}_{k}$ is $2 N$-by-4 matrix. When $1 \leq i \leq N, \mathbf{H}_{k}(i, j)=\frac{\partial \tau_{i}}{\partial \mathbf{x}_{k}(j)}$, otherwise $\mathbf{H}_{k}(i, j)=\frac{\partial f_{i-N}}{\partial \mathbf{x}_{k}(j)}$. Therefore, when $1 \leq i \leq N$, we have:

$$
\begin{aligned}
& \mathbf{H}_{k}(i, 1)=\frac{x}{\sqrt{x^{2}+y^{2}}}+\frac{x-x_{i, k}}{\sqrt{\left(x_{i, k}-x\right)^{2}+\left(y_{i, k}-y\right)^{2}}} \\
& \mathbf{H}_{k}(i, 2)=\frac{y}{\sqrt{x^{2}+y^{2}}}+\frac{y-y_{i, k}}{\sqrt{\left(x_{i, k}-x\right)^{2}+\left(y_{i, k}-y\right)^{2}}} \\
& \mathbf{H}_{k}(i, 3)=\mathbf{H}_{k}(i, 4)=0 .
\end{aligned}
$$

When $N+1 \leq i \leq 2 N$, we have the expression for $\mathbf{H}_{k}$ given in equation (22). 


$$
\begin{aligned}
\mathbf{H}_{k}(i, 1)= & \frac{V_{x}}{\sqrt{x^{2}+y^{2}}}-\frac{\left(V_{x} \cdot x+V_{y} \cdot y\right) \cdot x}{\left(\sqrt{x^{2}+y^{2}}\right)^{3}}+\frac{V_{x}-V \cdot \cos \psi_{i-N, k}}{\sqrt{\left(x_{i-N, k}-x\right)^{2}+\left(y_{i-N, k}-y\right)^{2}}}-\left(x-x_{i-N, k}\right) . \\
& \frac{\left(V_{x}-V \cos \psi_{i-N, k}\right)\left(x-x_{i-N, k}\right)+\left(V_{y}-V \sin \psi_{i-N, k}\right)\left(y-y_{i-N, k}\right)}{\left(\sqrt{\left(x-x_{i-N, k}\right)^{2}+\left(y-y_{i-N, k}\right)^{2}}\right)^{3}} \\
\mathbf{H}_{k}(i, 2)= & \frac{V_{y}}{\sqrt{x^{2}+y^{2}}}-\frac{\left(V_{x} \cdot x+V_{y} \cdot y\right) \cdot y}{\left(\sqrt{x^{2}+y^{2}}\right)^{3}}+\frac{V_{y}-V \cdot \sin \psi_{i-N, k}}{\sqrt{\left(x_{i-N, k}-x\right)^{2}+\left(y_{i-N, k}-y\right)^{2}}}-\left(y-y_{i-N, k}\right) . \\
& \frac{\left(V_{x}-V \cos \psi_{i-N, k}\right)\left(x-x_{i-N, k}\right)+\left(V_{y}-V \sin \psi_{i-N, k}\right)\left(y-y_{i-N, k}\right)}{\left(\sqrt{\left(x-x_{i-N, k}\right)^{2}+\left(y-y_{i-N, k}\right)^{2}}\right)^{3}} \\
\mathbf{H}_{k}(i, 3)= & \mathbf{H}_{k}(i-N, 1) \\
\mathbf{H}_{k}(i, 4)= & \mathbf{H}_{k}(i-N, 2) .
\end{aligned}
$$

In the expression for $\mathbf{H}_{k}, x, y, V_{x}, V_{y}$ are the position and velocity of the target at time $t=k$, and $x_{i, k}, y_{i, k}, \psi_{i, k}$ are the position and heading for the $i^{t h} \mathrm{UAV}$ at time $t=k$. After plugging (16) and (17) in, we take the derivative of $\mathbf{H}_{k}$ with respect to $\psi_{i, k}$. When $1 \leq i \leq N$, we have equation (23), otherwise we have equation (24), where

$$
\begin{aligned}
d_{k, x}^{i} & =x-x_{i, k-1}-V \cos \psi_{i, k} \Delta_{t} \\
d_{k, y}^{i} & =y-y_{i, k-1}-V \sin \psi_{i, k} \Delta_{t} \\
R_{k}^{i} & =\sqrt{\left(d_{k, x}^{i}\right)^{2}+\left(d_{k, y}^{i}\right)^{2}} \\
s_{k, x}^{i} & =V_{x}-V \cos \psi_{i, k} \\
s_{k, y}^{i} & =V_{y}-V \sin \psi_{i, k} .
\end{aligned}
$$

In practical scenarios, the UAV dynamics limit the rate at which its heading may change. Therefore, for each step we restrict the UAVs' position to an arc confined by the previous heading. In particular, we append the following constraint to the to optimization problem:

$$
\left|\psi_{i, k}-\psi_{i, k-1}\right| \leq C \forall i=1 \ldots N,
$$

where $C$ is the upper bound on the turning rate of the UAV.

\section{B. Measurement Entropy Maximization Approach}

In this section, we present an alternative approach based on maximizing the information or entropy introduced at each UAV measurement update. If we recall equation (4) and linearize around the approximate state and measurement vectors, we have:

$$
\mathbf{z}_{k} \approx \tilde{\mathbf{z}}_{k}+\mathbf{H}_{k}\left(\mathbf{x}_{k}-\tilde{\mathbf{x}}_{k}\right)+\boldsymbol{\nu}_{k}
$$

where $\tilde{\mathbf{x}}_{k}=\mathbf{A} \hat{\mathbf{x}}_{k-1}$ and $\tilde{\mathbf{z}}_{k}=\mathbf{h}_{k}\left(\tilde{\mathbf{x}}_{k}\right)$. If we define $\tilde{e}_{\mathbf{x}_{k}} \equiv$ $\mathbf{x}_{k}-\tilde{\mathbf{x}}_{k}$ and $\tilde{e}_{\mathbf{z}_{k}} \equiv \mathbf{z}_{k}-\tilde{\mathbf{z}}_{k}$, equation (26) can be simplified as:

$$
\tilde{e}_{\mathbf{z}_{k}}=\mathbf{H}_{k} \tilde{e}_{\mathbf{x}_{k}}+\boldsymbol{\nu}_{k}
$$

where $\tilde{e}_{\mathbf{z}_{k}}$ represents the new information our EKF can use to improve the estimate. We know:

$$
\begin{aligned}
E\left(\tilde{e}_{\mathbf{z}_{k}} \tilde{e}_{\mathbf{z}_{k}}^{H}\right) & =\mathbf{H}_{k} E\left(\tilde{e}_{\mathbf{x}_{k}} \tilde{e}_{\mathbf{x}_{k}}^{H}\right) \mathbf{H}_{k}^{H}+\mathbf{R}_{k} \\
& =\mathbf{H}_{k} \mathbf{P}_{k}^{-} \mathbf{H}_{k}^{T}+\mathbf{R}_{k},
\end{aligned}
$$

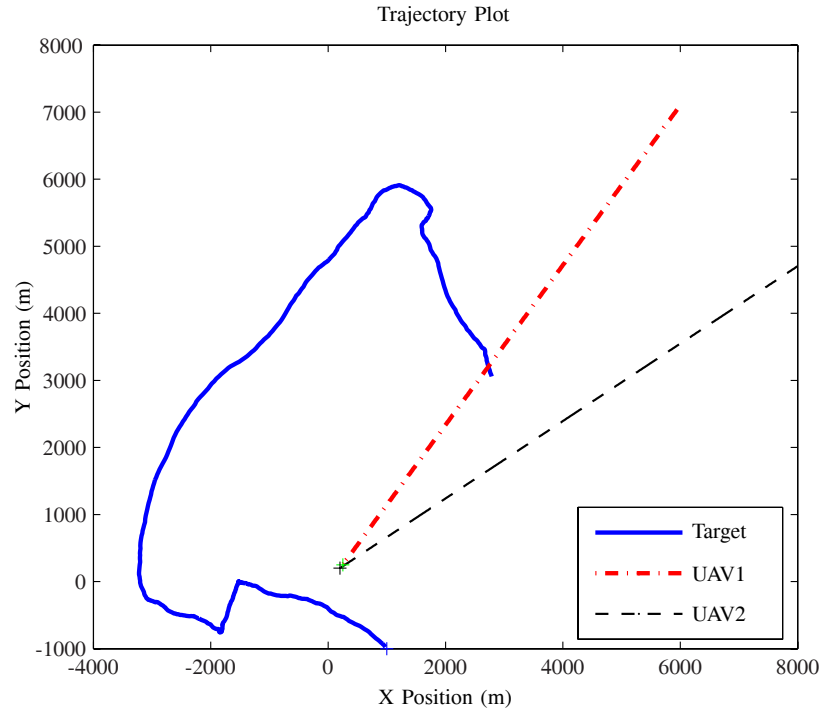

Fig. 1. No Control Case : UAV Trajectory

so the entropy of the new measurement is therefore:

$$
h\left(\tilde{e}_{\mathbf{z}_{k}}\right)=\frac{1}{2} \ln \left((2 \pi e)^{2 N}\left|E\left(\tilde{e}_{\mathbf{z}_{k}} \tilde{e}_{\mathbf{z}_{k}}^{H}\right)\right|\right)
$$

After getting rid of the constant term in (29), we simply need to maximize $\left|E\left(\tilde{e}_{\mathbf{z}_{k}} \tilde{e}_{\mathbf{z}_{k}}^{H}\right)\right|$. We refer to this approach as Measurement Entropy Maximization (MEM):

$$
\Psi_{k}=\underset{\Psi_{k}}{\arg \max }\left|\mathbf{H}_{k} \mathbf{P}_{k}^{-} \mathbf{H}_{k}^{T}+\mathbf{R}_{k}\right| .
$$

In our implementation, constraint (25) is taken into account, and the simple gradient search approach is used to find the optimum. While the global optimum is not always guaranteed, it yields excellent results in our simulations.

\section{Simulation Results}

In this section, we present simulation results for the OneStep-Ahead and MEM algorithms proposed above. Two UAVs are assumed to be tracking a target that moves according to the perturbed constant velocity model of (3). The nominal velocity of the target in the $x$ and $y$ directions is $V_{x}=-15 \mathrm{~m} / \mathrm{s}$ and 


$$
\begin{gathered}
\frac{\partial \mathbf{H}_{k}(i, 1)}{\partial \psi_{i, k}}=-d_{k, x}^{i} \cdot \frac{\left(d_{k, x}^{i} \cdot V \cdot \sin \psi_{i, k} \cdot \Delta_{t}-d_{k, y}^{i} \cdot V \cdot \cos \psi_{i, k} \cdot \Delta_{t}\right)}{\left(R_{k}^{i}\right)^{3}}+\frac{V \cdot \sin \psi_{i, k} \cdot \Delta_{t}}{R_{k}^{i}} \\
\frac{\partial \mathbf{H}_{k}(i, 2)}{\partial \psi_{i, k}}=-d_{k, y}^{i} \cdot \frac{\left(d_{k, x}^{i} \cdot V \cdot \sin \psi_{i, k} \cdot \Delta_{t}-d_{k, y}^{i} \cdot V \cdot \cos \psi_{i, k} \cdot \Delta_{t}\right)}{\left(R_{k}^{i}\right)^{3}}-\frac{V \cdot \cos \psi_{i, k} \cdot \Delta_{t}}{R_{k}^{i}} \\
\frac{\partial \mathbf{H}_{k}(i, 3)}{\partial \psi_{i, k}}=0 \\
\frac{\partial \mathbf{H}_{k}(i, 4)}{\partial \psi_{i, k}}=0
\end{gathered}
$$

$$
\begin{gathered}
\frac{\partial \mathbf{H}_{k}(i, 1)}{\partial \psi_{i-N, k}}=\frac{V \cdot \sin \psi_{i-N, k}}{R_{k}^{i-N}}-\frac{s_{k, x}^{i-N} \cdot\left(d_{k, x}^{i-N} \cdot V \cdot \sin \psi_{i-N, k} \Delta_{t}-d_{k, y}^{i-N} \cdot V \cdot \cos \psi_{i-N, k} \Delta_{t}\right)}{\left(R_{k}^{i-N}\right)^{3}} \\
-\frac{\left(d_{k, x}^{i-N} \cdot V \cdot \sin \psi_{i-N, k}+s_{k, x}^{i-N} \cdot V \cdot \sin \psi_{i-N, k} \Delta_{t}-d_{k, y}^{i-N} \cdot V \cdot \cos \psi_{i-N, k}-s_{k, y}^{i-N} \cdot V \cdot \cos \psi_{i-N, k} \Delta_{t}\right) d_{k, x}^{i-N}}{\left(R_{k}^{i-N}\right)^{3}} \\
+3 \cdot \frac{\left(s_{k, x}^{i-N} d_{k, x}^{i-N}+s_{k, y}^{i-N} d_{k, y}^{i-N}\right) d_{k, x}^{i-N} \cdot\left(d_{k, x}^{i-N} \cdot V \cdot \sin \psi_{i-N, k} \Delta_{t}-d_{k, y}^{i-N} \cdot V \cdot \cos \psi_{i-N, k} \Delta_{t}\right)}{\left(R_{k}^{i-N}\right)^{5}}-\frac{s_{k, x}^{i-N} d_{k, x}^{i-N}+s_{k, y}^{i-N} d_{k, y}^{i-N}}{\left(R_{k}^{i-N}\right)^{3}} \cdot V \cdot \sin \psi_{i-N, k} \Delta_{t} \\
\frac{\partial \mathbf{H}_{k}(i, 2)}{\partial \psi_{i-N, k}}=-\frac{V \cdot \cos \psi_{i-N, k}}{R_{k}^{i-N}}-\frac{s_{k, y}^{i-N} \cdot\left(d_{k, x}^{i-N} \cdot V \cdot \sin \psi_{i-N, k} \Delta_{t}-d_{k, y}^{i-N} \cdot V \cdot \cos \psi_{i-N, k} \Delta_{t}\right)}{\left(R_{k}^{i-N}\right)^{3}} \\
-\frac{\left(d_{k, x}^{i-N} \cdot V \cdot \sin \psi_{i-N, k}+s_{k, x}^{i-N} \cdot V \cdot \sin \psi_{i-N, k} \Delta_{t}-d_{k, y}^{i-N} \cdot V \cdot \cos \psi_{i-N, k}-s_{k, y}^{i-N} \cdot V \cdot \cos \psi_{i-N, k} \Delta_{t}\right) \cdot d_{k, y}^{i-N}}{\left(R_{k}^{i-N}\right)^{3}} \\
+3 \cdot \frac{\left(s_{k, x}^{i-N} d_{k, x}^{i-N}+s_{k, y}^{i-N} d_{k, y}^{i-N}\right) d_{k, y}^{i-N} \cdot\left(d_{k, x}^{i-N} \cdot V \cdot \sin \psi_{i-N, k} \Delta_{t}-d_{k, y}^{i-N} \cdot V \cdot \cos \psi_{i-N, k} \Delta_{t}\right)}{\left(R_{k}^{i-N}\right)^{5}}+\frac{s_{k, x}^{i-N} d_{k, x}^{i-N}+s_{k, y}^{i-N} d_{k, y}^{i-N}}{\left(R_{k}^{i-N}\right)^{3}} \cdot V \cdot \cos \psi_{i-N, k} \Delta_{t} \\
\frac{\partial \mathbf{H}_{k}(i, 3)}{\partial \psi_{i-N, k}}=\frac{\partial \mathbf{H}_{k}(i-N, 1)}{\partial \psi_{i-N, k}} \\
\frac{\partial \mathbf{H}_{k}(i, 4)}{\partial \psi_{i-N, k}}=\frac{\partial \mathbf{H}_{k}(i-N, 2)}{\partial \psi_{i-N, k}}
\end{gathered}
$$

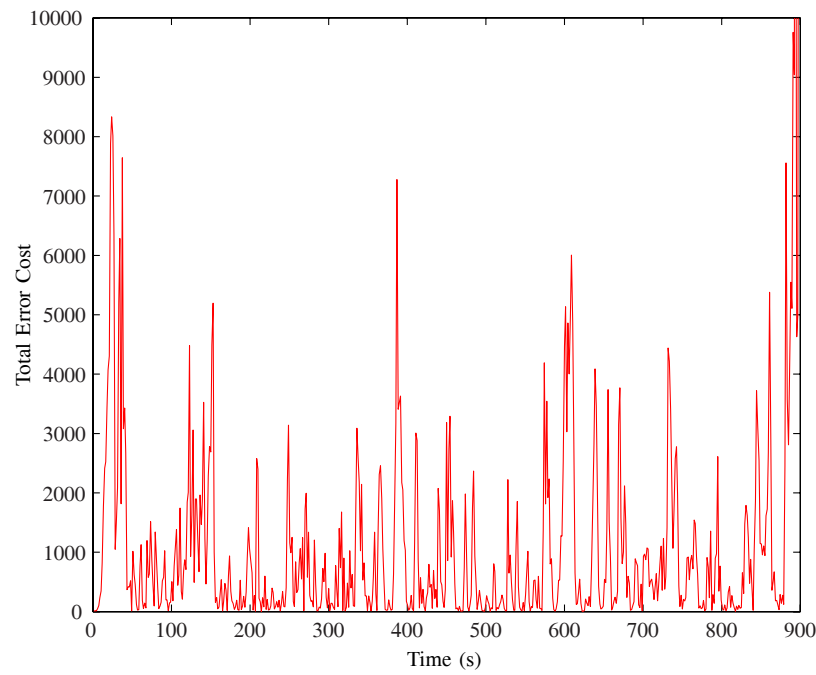

Fig. 2. No Control Case : Real Estimation Error

$V_{y}=15 \mathrm{~m} / \mathrm{s}$, and the initial target position is assumed to be at $(x, y)=(1000,-1000)$ meters. The two UAVs are initially positioned at $(250,250)$ and $(200,200)$ with headings $\frac{5 \pi}{18}$ and $\frac{\pi}{6}$, respectively. We also assume that each UAV flies at a constant speed of $V=10(\mathrm{~m} / \mathrm{s})$. In the simulation, the process covariance matrix $\mathbf{Q}_{k}$ is set to be $\operatorname{diag}(10,10,3,3)$ and the measurement covariance matrix $\mathbf{R}_{k}$ to be $\operatorname{diag}(80,80,10,10)$. Simulations are run for 900 seconds.

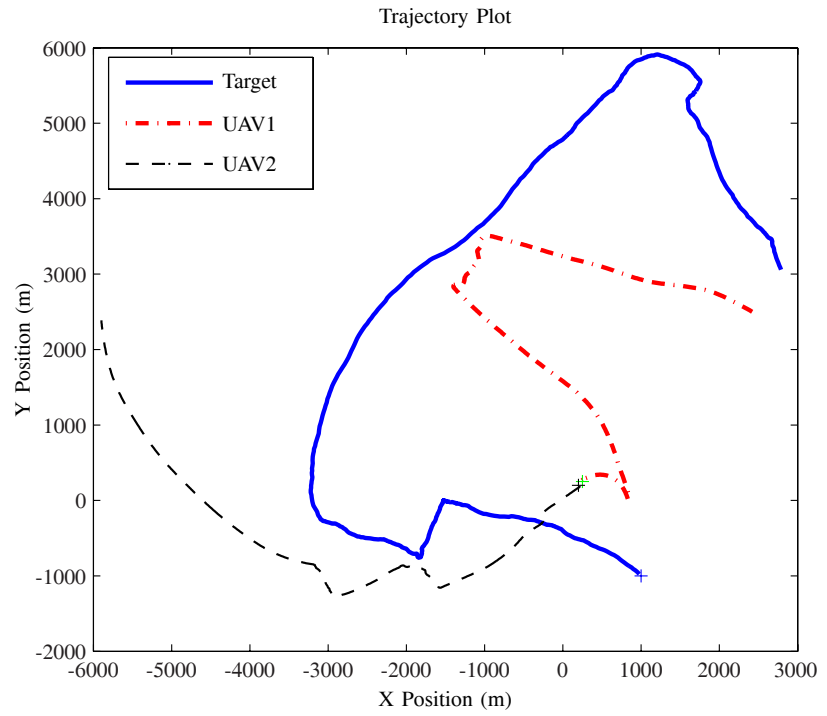

Fig. 3. One Step Ahead Case : UAV Trajectory

In Figure (1) and (2), we first show the case where the UAVs are not controlled. Figure (1) shows the paths of the target and uncontrolled UAVs, and Figure (2) shows the estimation error $\left\|\mathbf{x}_{k}-\hat{\mathbf{x}}_{k}\right\|^{2}$. Clearly, the tracking performance in this case is rapidly deteriorating. The performance of the constrained One-Step-Ahead approach is shown in Figure (3) and (4), and that of the MEM algorithm in Figure (5) and (6). Both show 


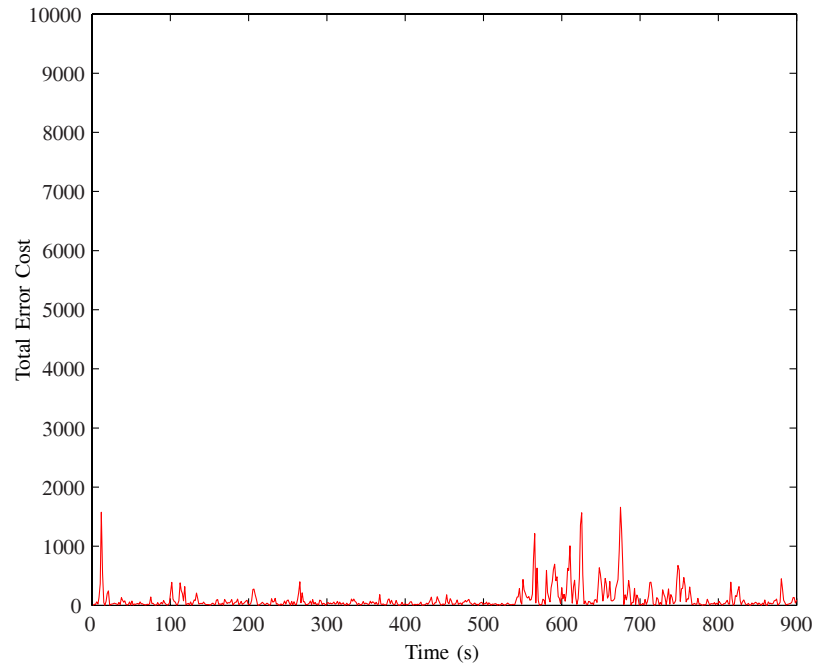

Fig. 4. One Step Ahead Case : Real Estimation Error

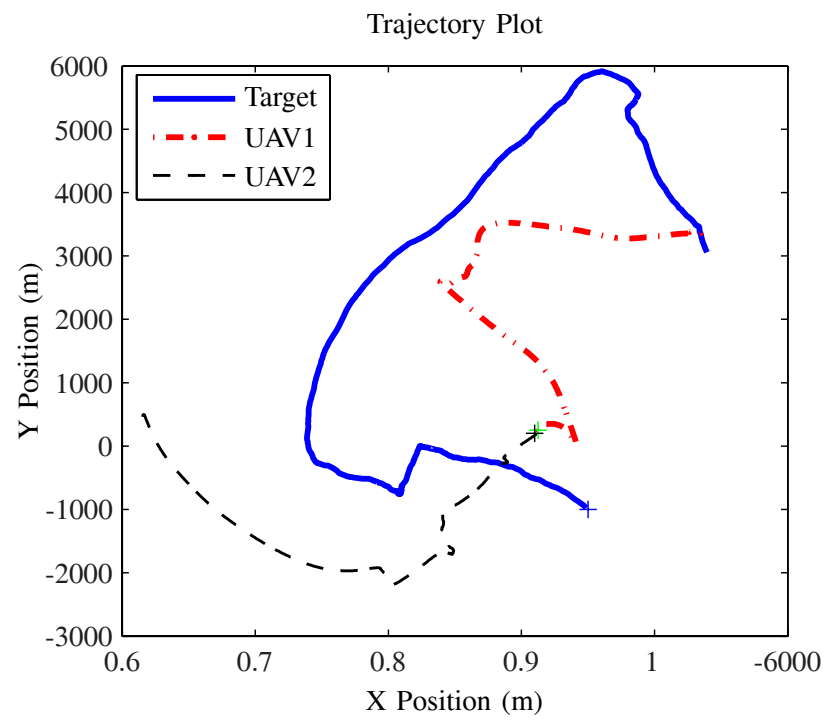

Fig. 5. Maximize Entropy Case : UAV Trajectory

essentially identical results that are significantly better than the uncontrolled EKF. Similar performance improvements were observed in many other cases as well, although the results show wide variations depending on the relative speed of the target and UAVs as well as their initial positions and headings.

\section{Conclusions}

In this paper, we presented two centralized control algorithms for optimally guiding the headings of UAVs employed in a target tracking application. The UAV headings are chosen either to maximize the measurement entropy or to minimize the trace of the prediction error covariance associated with an EKF tracker. By introducing an outer loop control over the EKF in this way, dramatically improved tracking results are achieved.

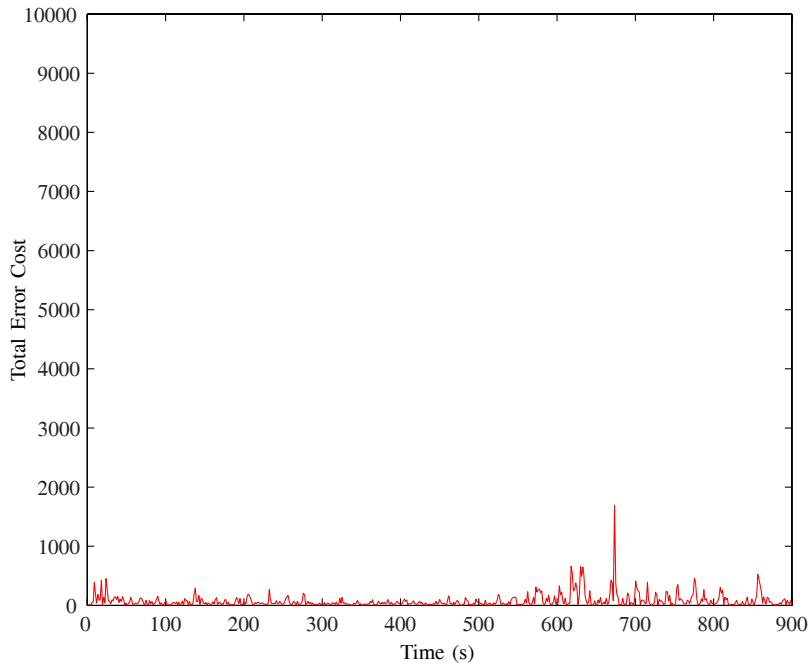

Fig. 6. Maximize Entropy Case : Real Estimation Error

\section{REFERENCES}

[1] G. Gu, P.R. Chandler, C.J. Schumacher, A. Sparks, and M. Prachter. Optimum cooperative uav sensing based on cramer-rao bound. In Proceedings of the American Control Conference, pages 4090-4095, Portland, Oregon, June 2005.

[2] B. Grocholsky, A. Makarenko, and H. Durrant-Whyte. Informationtheoretic coordinated control of multiple sensor platforms. In Proceedings of the IEEE International Conference on Robotics and Automation, volume 1, pages 1521-1526, 2003.

[3] Marcel L. Hernandez. Optimal sensor trajectories in bearings-only tracking. In Per Svensson and Johan Schubert, editors, Proceedings of the Seventh International Conference on Information Fusion, volume II, pages 893-900, Mountain View, CA, Jun 2004. International Society of Information Fusion.

[4] Y. Oshman and P. Davidson. Optimization of observer trajectories for bearings-only target localization. IEEE Trans. Aerosp. Electron. Syst., 35(3):892-902, July 1999.

[5] J. Ousingsawat and M. Campbell. Establishing trajectories for multivehicle reconnaissance. In Proceedings of the AIAA Guidance, Navigation, and Control Conference, pages 2188-2199, Aug. 2004.

[6] Ying He and K.P. Chong. Sensor scheduling for target tracking in sensor networks. In Proceedings of the IEEE Conference on Decision and Control, pages 743-748, December 2004.

[7] Randal et al. Beard. Autonomous vehicle technologies for small fixed wing uavs. AIAA Journal of Aerospace Computing, Information, and Communication, 2(1):92-108, 2005.

[8] Nicholas J. Willis. Bistatic Radar. Artech House, Boston, 1991. 